ISSN 0103-5150

Fisioter. Mov., Curitiba, v. 25, n. 3, p. 669-678, jul./set. 2012 Licenciado sob uma Licença Creative Commons

\title{
Efeito da eletroestimulação no músculo desnervado de animais: revisão sistemática
}

\author{
The effect of electrical stimulation on animals \\ denervated muscle: a systematic review
}

\section{Emanuela Virginia Vale Cavalcante ${ }^{[a]}$, Lívia Gabriely Melo da Silva ${ }^{[b]}$, Eduardo José Nepomuceno Montenegro ${ }^{[\mathrm{cl}}$, Nicodemos Tele de Pontes Filho ${ }^{[\mathrm{d}]}$}

[a] Fisioterapeuta, mestre pelo programa de Pós-Graduação em Fisioterapia da Universidade Federal de Pernambuco (UFPE), Recife, PE - Brasil, e-mail: emanuela.vale@gmail.com

[b] Discente do curso de Fisioterapia da Universidade Federal de Pernambuco (UFPE), Recife, PE - Brasil, e-mail: livinha_gaby@hotmail.com

[c] Docente do Departamento de Fisioterapia da Universidade Federal de Pernambuco (UFPE), Recife, PE - Brasil, e-mail: eduardo.montenegro@ufpe.br

[d] Docente do Programa de Pós-Graduação em Fisioterapia da Universidade Federal de Pernambuco (UFPE), Recife, PE Brasil, e-mail: ntpf@ig.com.br

\section{Resumo}

Introdução: A recuperação funcional após a lesão nervosa periférica está relacionada a fatores intrínsecos e extrínsecos ao sistema nervoso periférico, tais como a gravidade da lesão e a condição dos órgãos-alvo. A atrofia constitui uma das principais alterações do músculo após a lesão nervosa e, uma vez instalada, atua como barreira ao crescimento axonal durante a reinervação muscular. 0 uso da eletroestimulação é rotineiro no campo da fisioterapia e tem o objetivo de minimizar ou impedir a atrofia muscular e, assim, favorecer a recuperação da lesão nervosa periférica. Objetivo: Avaliar os efeitos da eletroestimulação sobre as características tróficas do músculo desnervado. Métodos: Artigos publicados entre 1990 e 2010 e indexados aos bancos de dados da PUBMED foram selecionados utilizando os seguintes descritores: "muscle denervation AND electric stimulation" e "muscular atrophy AND electric stimulation". Foram considerados como critério de inclusão os estudos experimentais em animais (ratos) que utilizassem a lesão nervosa periférica como modelo de desnervação e que avaliassem o efeito da eletroestimulação muscular sobre a área de secção transversa e/ou a massa muscular de músculos desnervados. Resultados: Nove artigos foram selecionados 
para a revisão. Conclusões: 0 efeito da eletroestimulação está diretamente relacionado à característica do protocolo de intervenção, que, quando aplicado de maneira adequada, apresenta o efeito de retardar e, em alguns casos, impedir a atrofia do músculo desnervado.

Palavras-chave: Desnervação muscular. Estimulação elétrica. Atrofia muscular.

\begin{abstract}
Introduction: The functional recovery after peripheral nerve injury is related to extrinsic and intrinsic factors of peripheral nervous system, like trauma severity and conditions of the end-organs. The atrophy is a major change of muscles after nerve injury, and once installed, it acts as a barrier to axonal growth during muscle reinnevation. The use of electrical stimulation is routine in the field of physical therapy and aims to minimize or prevent muscle atrophy and facilitate recovery from peripheral nerve injury. Objective: The aim of this review is to evaluate the effects of electrical stimulation on tropics characteristics of denervated muscle. Methods: Articles published between 1990 and 2010 from periodicals indexed in the PUBMED database were selected, using the following keywords: "muscle denervation AND electric stimulation" and "muscular atrophy AND electric stimulation". Were included experimental studies in animals (rats) that had used a peripheral nerve lesion as a model of muscle denervation and that had evaluated the effect of electrical muscle stimulation on cross-sectional area and/or muscle mass weight of denervated muscles. Results: 9 articles were selected for this review. Conclusion: The effect of electrical stimulation is directly related to the intervention protocol's characteristics, which when applied properly had the effect of delaying, in some cases, or prevents the atrophy of denervated muscle.
\end{abstract}

Keywords: Muscle denervation. Electric stimulation. Muscular atrophy.

\section{Introdução}

A lesão nervosa periférica é uma ocorrência comum na prática clínica de fisioterapia. Em geral, é decorrente de traumas, como de acidentes veiculares, com objetos penetrantes, por quedas e acidentes industriais (1).

O comprometimento na transmissão dos impulsos nervosos é uma das consequências desse tipo de lesão nervosa (2), que conduz a diversas alterações no sistema muscular, tais como atrofia muscular $(3,4)$, proliferação do tecido conjuntivo (TC) (5), mudanças na excitabilidade muscular (6) e modificações nas células satélites $(7,8)$.

A perda do suprimento nervoso promove a diminuição da atividade muscular, que, por sua vez, desencadeia uma resposta adaptativa para a remodelação do tecido muscular, marcada pela degradação das proteínas contráteis do músculo via sistema proteolítico $(9,10)$. Como resultado, observa-se diminuições da massa $(5,11)$, do diâmetro da fibra (12) e da produção de força $(13,14)$, as quais, em conjunto, caracterizam a atrofia do músculo.

Essas modificações iniciam imediatamente após a desnervação e persistem enquanto o músculo estiver desprovido de inervação. Caso a desnervação permaneça por um longo período de tempo, o TC substituirá os elementos contráteis do músculo, submetidos à proteólise, e atuará como inibidor da regeneração muscular após a reinervação $(11,15)$.

O prognóstico das lesões do nervo periférico é variável e depende da gravidade delas. Nos casos em que há preservação da estrutura mesenquimal, seja ela dada por reparo cirúrgico ou pelo próprio trauma, o prognóstico é favorável, pois a presença dessa estrutura intacta serve como guia para o crescimento axonal até sua reinervação com órgão-alvo (16-19).

No entanto, apesar da capacidade regenerativa do sistema nervoso periférico, a recuperação após a lesão nem sempre é completa e satisfatória, em virtude de fatores intrínsecos e extrínsecos ao sistema nervoso periférico, como o tipo e o nível de lesão, a presença de patologias associadas, além de alterações nos órgãos-alvo $(20,21)$.

A condição do músculo no momento da reinervação é determinante na recuperação funcional, tendo em vista que o músculo atrofiado atua como barreira ao crescimento axonal e impede que o desenvolvimento e a maturação axonal ocorram (9). Dessa 
forma, é comum, na prática clínica da fisioterapia, o emprego da eletroestimulação com o objetivo de minimizar a atrofia muscular e favorecer boa recuperação funcional da lesão nervosa periférica (22-25).

Nesse contexto, o presente estudo tem por objetivo realizar uma revisão sistemática dos estudos experimentais, a fim de verificar o efeito trófico da eletroestimulação em músculos desnervados.

\section{Materiais e métodos}

Artigos publicados entre 1990 e 2010 e indexados nos bancos de dados da PUBMED foram selecionados para este trabalho de revisão. A estratégia de busca utilizada foi: "muscle denervation AND electric stimulation" e "muscular atrophy AND electric stimulation", sendo inclusos, também, os seguintes limites: animals, english, spanish e portuguese. 0 período de busca dos artigos ocorreu entre $1^{\circ}$ de novembro de 2010 e 22 de novembro de 2010.

A seleção dos artigos foi realizada por dois avaliadores independentes e, no caso de discordâncias, um terceiro examinador foi convocado para o consenso final. Inicialmente, foram analisados os títulos e os resumos dos artigos, e aqueles considerados como potencialmente relevantes, segundos os critérios de inclusão e exclusão, foram adquiridos em sua versão completa para análise mais criteriosa.

Foram incluídos estudos experimentais em animais, especificamente os que utilizaram ratos, os quais tivessem a lesão nervosa periférica como modelo de desnervação e que avaliassem o efeito da eletroestimulação (EE) muscular sobre a área de secção transversa e/ou a massa muscular desses músculos nos períodos de desnervação.

Foram excluídos os estudos em que o emprego da EE ocorresse, também ou exclusivamente, no período de reinervação muscular, pois, nesse caso, não há a possibilidade de diferenciar o efeito trófico promovido pela EE daquele proporcionado pelo retorno do suprimento nervoso.

Foram também excluídos os estudos que fizessem uso da eletroestimulação associada a terapias com células-tronco e fármacos, pois o uso concomitante de outra terapia pode mascarar o efeito trófico da EE, e os que não descrevessem os dados concernentes ao procedimento de lesão, bem como os do protocolo de intervenção.

\section{Resultados}

Dentre os 254 artigos identificados inicialmente na busca eletrônica, apenas 12 foram obtidos em sua versão completa para uma análise mais detalhada, e, entre eles, dois foram excluídos por avaliar o efeito da EE durante a fase de reinevervação e um por não fornecer informações acerca da metodologia de lesão e da intervenção (Figura 1).

Dessa forma, segundo os critérios de inclusão e exclusão, nove artigos foram selecionados para a revisão: Gigo-Benato et al. (26); Tomori et al. (27); Russo et al. (28); Lima et al. (29); Russo et al. (30); Dow et al. (31); Dow et al. (32); Dow et al. (33); e Kanaya et al. (34).

Em todos os estudos selecionados, o planejamento de intervenção foi realizado pela combinação de aspectos temporais, como duração (período de tempo por dia), frequência (número de sessões por semana) e período (quantidade de semanas), com aspectos específicos da eletroestimulação, que incluem parâmetros da amplitude, frequência, duração do impulso, intervalo de repouso e número de contrações, conforme ilustrado no Quadro 1. Os resultados da análise dos desfechos de interesse - área de secção transversa e peso da massa muscular - estão apresentados no Quadro 2.

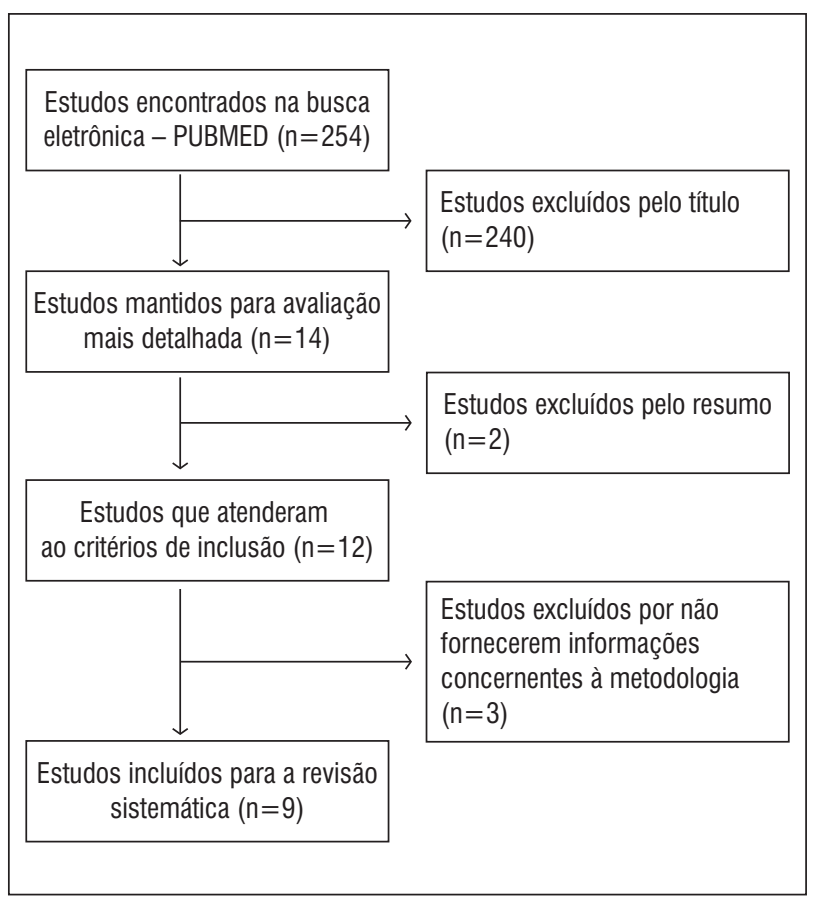

Figura 1 - Fluxograma da busca e seleção dos estudos para a revisão sistemática

Fonte: Dados da pesquisa. 


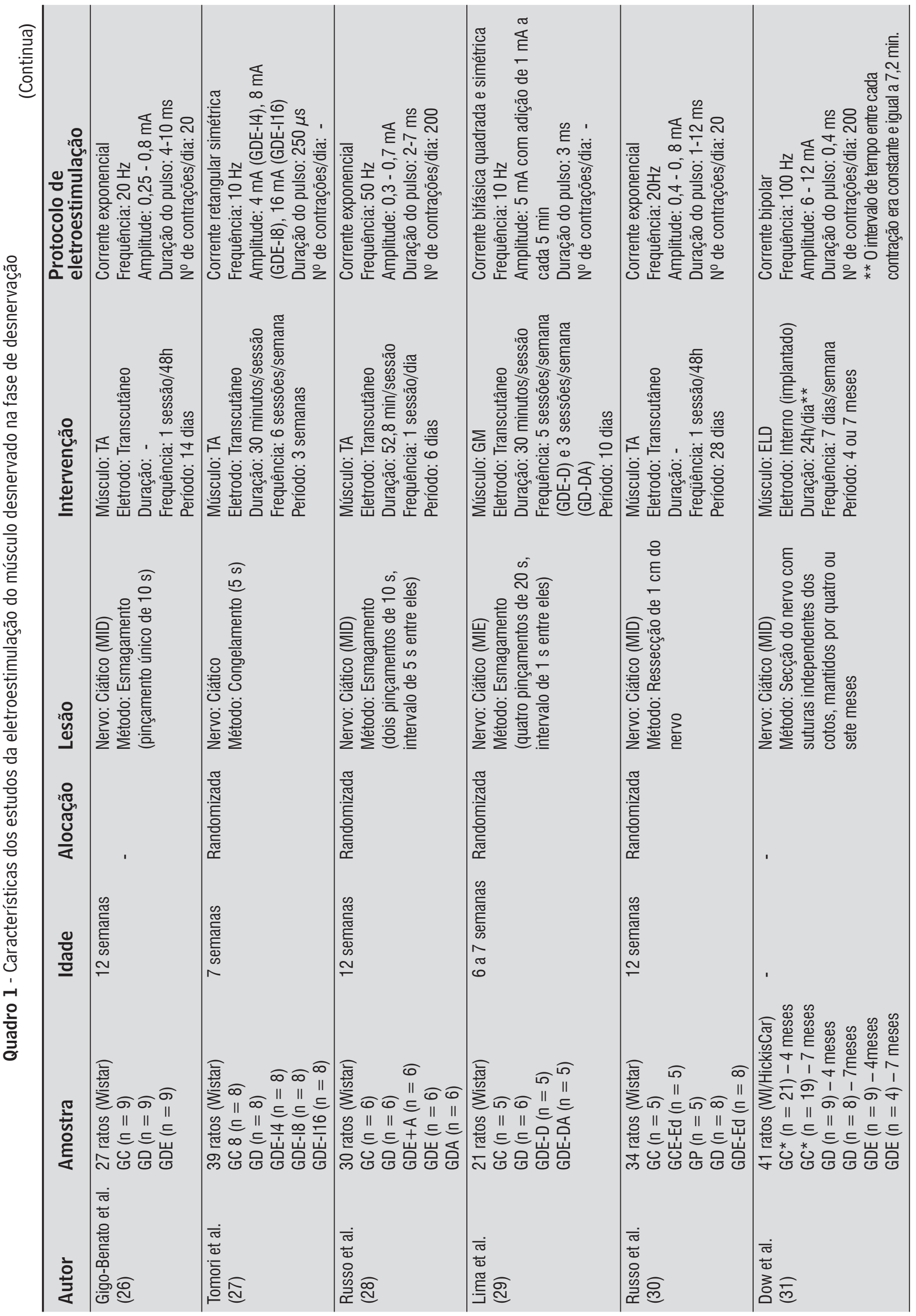




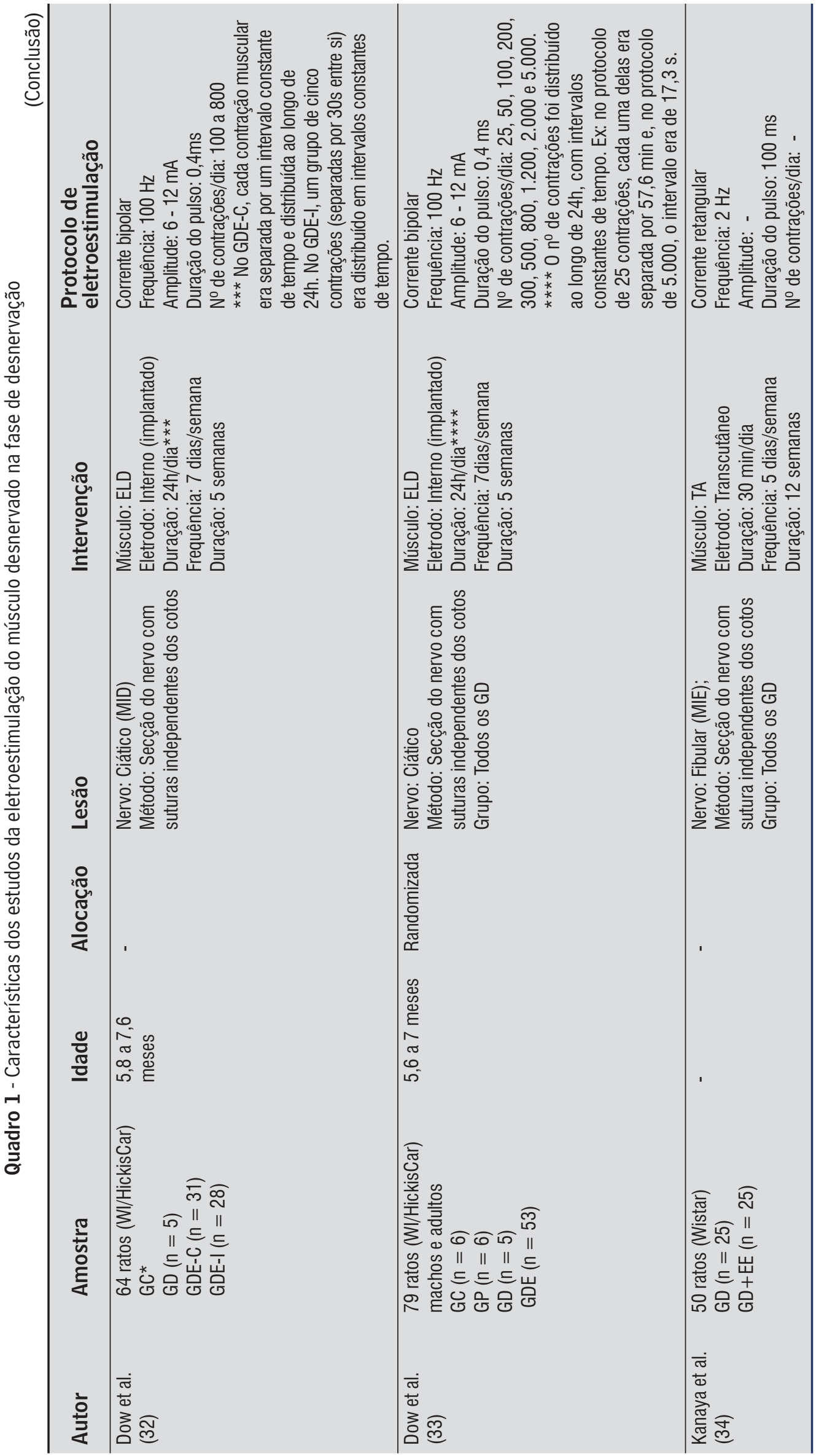

후을 틍 응 흔

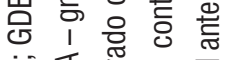

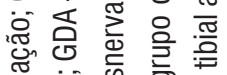

할

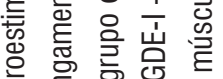

헝 흥 1 iٓ

E U ए ए

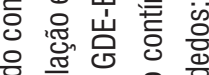

ग

을 要

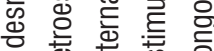

융 क क 은

흔 등 픙 홍

1 : E

峲 帝

ㄴ. 늃

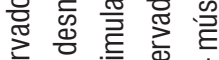

क्ष

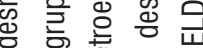

은 1 헝 을 을

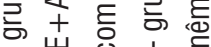

1 嵌 0

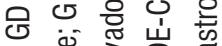

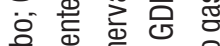

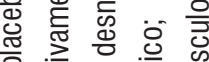

说 을

는 은 혼

1 更

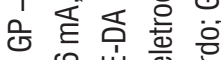

드응

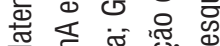

笨

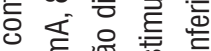

윽 함

फ 흘

ह 응

응 윰

응 훻을

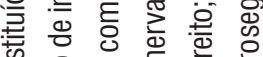

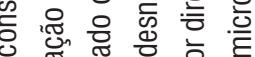

을 궣을

衰产离

잉

윽 훙을 둥

结

이일

* क्ञ

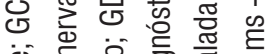

产 कू

क

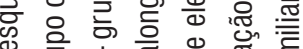

늘 1 त

중

0

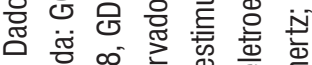

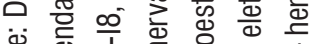

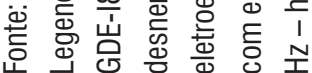


Quadro 2 - Efeitos da eletroestimulação nos grupos desnervados com eletroestimulação em relação aos grupos desnervados e controle

\begin{tabular}{|c|c|c|c|c|}
\hline \multirow[t]{2}{*}{ Autor } & \multicolumn{2}{|c|}{ Peso da massa muscular } & \multicolumn{2}{|c|}{ Área de secção transversa } \\
\hline & GDE $x$ GD & GDE $\times$ GC & GDE $\times$ GD & GDE x GC \\
\hline $\begin{array}{l}\text { Gigo-Benato } \\
\text { et al. } \\
\text { (26) }\end{array}$ & Nenhuma diferença & $\downarrow(p<0,05)$ & $\begin{array}{c}\downarrow \text { em } 55 \% \text { (GDE) e } 38 \% \text { (GD) } \\
(p<0,05)\end{array}$ & $\downarrow(p<0,05)$ \\
\hline $\begin{array}{l}\text { Tomori et al. } \\
(27)\end{array}$ & $\begin{array}{c}\uparrow \text { nos GDE-18 e I16 } \\
(p<0.05)\end{array}$ & $\downarrow(p<0,05)$ & - & - \\
\hline $\begin{array}{l}\text { Russo et al. } \\
\text { (28) }\end{array}$ & Nenhuma diferença & $\downarrow(p<0,05)$ & Nenhuma diferença & $\begin{array}{l}\downarrow \text { em 26\% (GDE), 25\% (GD) } \\
\text { e 24\% (GDE-A) (p < 0,05) }\end{array}$ \\
\hline $\begin{array}{l}\text { Lima et al. } \\
\text { (29) }\end{array}$ & Nenhuma diferença & $\downarrow(p=0,000)$ & Nenhuma diferença & $\begin{array}{c}\downarrow \text { em torno } 68 \% \text { em } \\
\text { todos os grupos com } \\
\text { eletroestimulação }(p= \\
0,000)\end{array}$ \\
\hline $\begin{array}{l}\text { Russo et al. } \\
(30)\end{array}$ & Nenhuma diferença & $\begin{array}{c}\downarrow \text { em } 62 \%(G D) \text { e } 66(G D E) \\
(p<0,05)\end{array}$ & & \\
\hline $\begin{array}{l}\text { Dow et al. } \\
\text { (31) }\end{array}$ & $\uparrow$ & $\downarrow(p<0,05)$ & - & - \\
\hline $\begin{array}{l}\text { Dow et al. } \\
\text { (32) }\end{array}$ & $\begin{array}{l}0 \text { GD manteve } \\
\text { apenas } 53 \% \text { do peso } \\
\text { muscular, e os GDE } \\
\text { mantiveram o peso } \\
\text { em torno de } 80 \text { e } \\
90 \% \text { em relação ao } \\
\text { GC. }\end{array}$ & $\begin{array}{l}\text { Nenhuma diferença, o peso } \\
\text { manteve-se em torno de } \\
90 \%(200 \text { a } 800 \text { Cd) e } 80 \% \\
\text { (GDE-C com } 100 \text { Cd). }\end{array}$ & - & - \\
\hline $\begin{array}{l}\text { Dow et al. } \\
\text { (33) }\end{array}$ & $\begin{array}{c}\uparrow, \text { exceto no GDE } \\
+25 \mathrm{Cd}(p<0,05)\end{array}$ & $\begin{array}{l}\text { Diferença apenas no GDE } \\
\text { com } 25 \mathrm{Cd}\end{array}$ & $\begin{array}{l}\text { Atenuou a redução nos } \\
\text { grupos de } 300 \text { a } 2.000 \mathrm{Cd} \text {, } \\
\text { sendo observado hipertrofia } \\
\text { no grupo com } 300 \mathrm{Cd} \text {. }\end{array}$ & $\downarrow(p<0,05)$ \\
\hline $\begin{array}{l}\text { Kanaya et al. } \\
\text { (34) }\end{array}$ & $\uparrow(p<0,05)$ & $\downarrow(p<0,05)$ & - & - \\
\hline
\end{tabular}

Fonte: Dados da pesquisa.

Legenda: GDE - grupo desnervado com eletroestimulação; GD - grupo desnervado; GC - grupo controle; GDE-C - grupo desnervado com eletroestimulação contínua; GDE + A - grupo desnervado com eletroestimulação e alongamento; Cd - número de contrações por dia.

\section{Discussão}

A eletroestimulação (EE) não é um recurso terapêutico novo, entretanto, algumas controvérsias acerca de sua influência sobre o trofismo de músculos desnervados ainda persistem, conforme demonstrado nos resultados desta revisão.

A literatura descreve que as mudanças tróficas de músculos desnervados podem ser resultantes de dois fatores: carência de fatores tróficos e atividade neuromuscular. Para alguns autores, as alterações morfológicas na desnervação são resultantes tanto da inatividade muscular quanto da ausência dos fatores tróficos. Assim, o desempenho insatisfatório da EE, apresentado em alguns trabalhos nesta revisão, seria justificado por ele suprir somente a ausência de um único fator, o neuromuscular $(35,36)$.

No entanto, ainda não há respaldo suficiente para garantir a influência dos fatores tróficos sobre as características morfológicas do músculo desnervado, visto que eles parecem estar mais relacionados à estabilização pós-sináptica da junção neuromuscular. De modo contrário, tem sido descrito que a atividade muscular é que desempenha o papel predominante, senão exclusivo sobre essas propriedades do músculo (37).

Dessa forma, considerando que não há evidência suficiente para demonstrar a influência dos fatores 
tróficos sobre a morfologia muscular, em que o fator neuromuscular parece ser soberano, a provável causa para os resultados contraditórios apresentados nesta revisão pode ser atribuída à variável metodologia dos estudos.

Dow et al. $(32,33)$ descrevem que o peso da massa de músculos desnervados e submetidos a EE apresentaram valores significativamente maiores ao de músculos desnervados sem intervenção e similares ao do músculo ileso. Dow et al. (33) descrevem, ainda, a preservação do diâmetro das fibras de músculos desnervados. Nesses estudos, os autores destacam que há uma relação direta entre o número de contrações geradas por dia e a distribuição delas com o efeito trófico da EE em músculos desnervados, sendo indicadas de 200 a 800 contrações/dia, com distribuição contínua e constante ao longo do dia.

Em contrapartida, a utilização do mesmo protocolo de intervenção proposto por Dow et al. (31), porém por um período mais prolongado de tempo, não proporcionou aumento significativo da massa muscular. Nesse caso, a divergência é explicada pelo defeito em algumas unidades do dispositivo gerador de EE, elaborado inicialmente por Dennis e Dow (38, 39) para uso em menor período de tempo, o qual, neste estudo, apresentou falhas na bateria.

Os autores, ao avaliar a correlação entre a falha do dispositivo e a massa muscular, observaram que, entre os animais que não necessitaram de reposição do dispositivo, o valor da massa muscular apresentou-se significativamente superior ao dos que realizaram reposição do aparelho e mais próximo aos músculos de animais do grupo controle. Na análise final dos dados, não houve distinção entre os que falharam ou não, o que torna questionável os dados desse trabalho.

Gigo-Benato et al. (26), Tomori et al. (27), Russo et al. (28, 30), Lima et al. (29) e Kanaya et al. (34) também propuseram verificar o efeito trófico da EE na fase de desnervação, mas, diferentemente dos trabalhos de Dow et al. $(31,33)$, esses autores empregaram um protocolo de intervenção não invasivo e com duração fásica menor do que oito horas por dia. Dentre esses, somente Tomori et al. (27) e Kanaya et al. (34) apontaram o efeito benéfico da EE em minimizar a atrofia muscular.

Tomori et al. (27) verificaram que o uso da corrente de baixa intensidade $(4 \mathrm{~mA})$ mostrou-se ineficaz em minimizar a atrofia muscular, ao contrário das correntes de maior intensidade (8 e $16 \mathrm{~mA}$ ), que foram efetivas em reduzir a atrofia do músculo desnervado. Outras variáveis também foram avaliadas nesse estudo, e os autores chamam a atenção para o efeito adverso da corrente de $16 \mathrm{~mA}$ sobre a regeneração dos terminais nervosos na placa motora. Assim, os autores concluem que a intensidade da corrente tem relação direta com os efeitos da EE e não recomendam intensidades muito baixas nem muito altas.

Kanaya et al. (34) constataram que um protocolo de EE com duração de pulso maior (igual a 100 ms) promoveu aumento significativo da massa muscular no grupo desnervado e tratado em relação ao grupo desnervado e não tratado.

Por outro lado, Gigo-Benato et al. (26) e Russo et al. (30) não relataram nenhum efeito da EE sobre o estado trófico dos músculos desnervados. A possível causa para esse resultado pode estar relacionado ao número de contrações geradas pelo protocolo, apenas vinte contrações por dia. Como explanado anteriormente, Dow et al. (33) descreveram a relação direta entre o efeito trófico da EE e o número de contrações por dia, sendo que o efeito trófico da EE só foi observado nos protocolos acima de cinquenta contrações por dia.

Nesse sentido, Russo et al. (28) elaboraram um novo estudo com o protocolo de intervenção semelhante ao estudo anterior (30), porém com o número de contrações/dia igual a 200, conforme recomendado por Dow et al. (33). Os resultados desse trabalho, no entanto, não demonstraram nenhum efeito benéfico da EE. Nesse caso, a baixa intensidade da corrente, que não ultrapassou $0,7 \mathrm{~mA}$, pode ser a causa desse resultado.

Lima et al. (29) também não observaram diferença nos valores da massa muscular e da área de secção transversa entre os grupos de intervenção e o de desnervação, resultado semelhante aos obtidos por Caierão et al. (15) e Fernandes et al. (5), para o uso do mesmo protocolo de intervenção.

Sabe-se que os valores de reobase e cronaxia conferem uma eficiente descrição da excitabilidade muscular e se apresentam mais elevados em músculos desnervados, demonstrando menor excitabilidade deles em relação ao músculo sadio (21). Graças a essa pouca excitabilidade, os músculos desnervados necessitam de maior amplitude (reobase) e duração de pulso (cronaxia) para se contrair $(40,41)$.

Dessa forma, os protocolos de EE em que os parâmetros da corrente elétrica correspondiam às características da baixa excitabilidade do músculo 
desnervado, com o emprego de amplitude (27) ou duração (34) e pulso maiores, foram os que se mostraram eficazes em minimizar a perda do trofismo em músculos desnervados.

Semelhantemente ao protocolo proposto por Kanaya et al. (34) e tendo em vista essa característica do músculo desnervado, Mokrusch et al. (42) aplicaram um protocolo com largura de pulso maior e duração menor da intervenção, no qual se verificou que o diâmetro das fibras musculares para o grupo tratado foi de 72 a $86 \%$ do controle. Nesse estudo, os autores sugerem que a maior duração de pulso pode ter contrabalanceado a menor duração da intervenção, justificativa provável para explicar os resultados de Kanaya et al. (34), em detrimento dos de Gigo-Benato et al. (26) , Russo et al. $(28,30)$ e Lima et al. (29).

De forma contrária pode ter ocorrido nos trabalhos de Dow et al. $(32,33)$, em comparação aos últimos citados, pois, embora tenham utilizado largura e intensidade de pulso semelhantes, somente os resultados descritos pelo primeiro autor foram favoráveis, em que a aplicação da EE por um período maior pode ter compensado a curta duração de pulso.

No que se refere à metodologia dos estudos, observou-se a omissão de dados relevantes do protocolo de EE, em particular, o número de contrações geradas por dia, o que, como demonstrado por Dow et al. (33), tem relação direta com o ganho de trofismo. Verificou-se, também, que o peso da massa muscular foi o desfecho mais avaliado entre os estudos.

A pesagem da massa é uma técnica simples, porém, reflete indiretamente o trofismo das fibras, visto que também é influenciada por outros fatores como a proliferação do tecido conjuntivo. Dessa forma, o uso dessa medida pode favorecer o mascaramento da atrofia e, nesse sentido, a análise histológica com a determinação da área de secção transversa parece ser mais confiável e indicada para avaliação do trofismo muscular (40).

\section{Conclusão}

Os estudos incluídos nesta revisão sistemática apresentaram variabilidade metodológica, que, por sua vez, pode ter influenciado na diversidade de resultados. Entretanto, aqueles que aplicaram um protocolo de EE adequado à característica de baixa excitabilidade do músculo desnervado, por meio do emprego de amplitude ou duração de pulso maior, bem como aqueles em que o uso da EE ocorreu por período suficiente para produzir no mínimo 50 contrações por dia, mostraram-se eficazes em minimizar a perda do trofismo em músculos desnervados.

Assim, pode-se afirmar que a eletroestimulação apresenta efeito trófico sobre os músculos desnervados, desde que os parâmetros da corrente atendam à baixa excitabilidade do músculo desnervado. E, por fim, recomenda-se, para estudos posteriores, mais detalhamento do protocolo de intervenção, bem como a utilização da área de secção transversa como parâmetro avaliativo do trofismo muscular.

\section{Referências}

1. Robinson LR. Traumatic injury to peripheral nerves. Muscle Nerve. 2000;23(6):863-73. doi:10.1002/ (SICI) 1097 - 4598(200006) 23:6<863: AID MUS4>3.0.CO;2-0.

2. Sunderland S. Nerve and nerve injure. 2nd ed. London: Churchill Livingstone; 1985.

3. Liu M, Zhang D, Shao C, Liu J, Ding F, Gu X. Expression pattern of myostatin in gastrocnemius muscle of rats after sciatic nerve crush injury. Muscle Nerve. 2007;35(5):649-56. doi:10.1002/mus.20749.

4. Hulataa D, Hughesa WF, Shott S, Kroinc JS, Gonzalezd MH, Kernsa JM. Early behavioral and histological outcomes following a novel traumatic partial nerve lesion. J Neurosci Methods. 2008;172(2):236-44. doi:10.1016/j.jneumeth.2008.05.002.

5. Fernandes KCBG, Polacow MLO, Guirro RRJ, Campos GER, Somazz MC, Pinto VF, et al. Análise morfométrica dos tecidos musculares e conjuntivo após desnervação e estimulação elétrica de baixa freqüência. Rev Bras Fisioter. 2005;9(2):235-41.

6. Russo TL, França CN, Castro CES, Salvini TF. Alterações da cronaxia, reobase e da acomodação no músculo esquelético desnervado submetido à eletroestimulação. Rev Bras Fisioter. 2004;8(2):169-75.

7. Kujawa M, Baran W, Jankowska-Steifer E. Quantitative ultrastructural changes in satellite cells of rats immobilized after soleus muscle denervation. Exp Mol Pathol. 2005;78(1):78-85. doi.org/10.1016/j. yexmp.2004.08.007. 
8. Schmalbruch H, Lewis DM. Dynamics of nuclei of muscle fibers and connective cells in normal and denervated rat muscles. Muscle Nerve. 2000;23(4):617-26. doi:10.1002/(SICI)1097-4598(200004)23:4<617::AIDMUS22>3.0.CO;2-Y.

9. Sultan KR, Dittrich BT, Pette D. Calpain activity in fast, slow, transforming, and regenerating skeletal muscles of rat. Am J Physiol Cell Physiol. 2000;279:C639-47.

10. Ferreira R, Neuparth MJ, Ascensão A, Magalhães J, Duarte J, Amado F. Atrofia muscular esquelética. Modelos experimentais, manifestações teciduais e fisiopatologia. RPCD. 2004;4(3):94-111.

11. Marqueste T, Decherchi P, Desplanches D, Favier R, Grelot L, Jammes Y. Chronic electrostimulation after nerve repair by self-anastomosis: effects on the size, the mechanical, histochemical and biochemical muscle properties. Acta Neuropathol. 2006;111(6):589600. doi:10.1007/s00401-006-0035-2.

12. Cole BG, Gardiner PF. Does electrical stimulation of denervated muscle, continued after reinnervation, influence recovery of contractile function? Exp Neurol. 1984;85(1):52-62. doi:10.1016/00144886(84)90159-6.

13. Aydin MA, Mackinnon SE, Gu XM, Kobayashi J, Kuzon WM. Force deficits in skeletal muscle after delayed reinnervation. Plast Reconstr Surg. 2004;113(6):17128. doi:10.1097/01.PRS.0000118049.93654.CA.

14. Minamoto VB. Efeitos da desnervação no músculo esquelético: uma revisão. Fisioter Mov. 2007;20(3):63-9.

15. Caierão QM, Betini J, Teodori RM, Minamoto VB. 0 efeito do intervalo da estimulação elétrica no músculo desnervado de rato. Rev Bras Fisioter. 2008;12(2):143-8.

16. Burnett MG, Zager EL. Pathophsiology of peripheral nerve injury: a brief review. Neurosurg Focus. 2004;16(5):1-7. doi:10.3171/foc.2004.16.5.2.

17. Siqueira R. Lesões nervosas periféricas: uma revisão. Rev Neurocienc. 2007;15(3):226-33.

18. Rodríguez FJ, Valero-Cabré A, Navarro X. Regeneration and functional recovery following peripheral nerve injury. Drug Discovery Today: Disease Models. 2004;1(2):177-85. doi:10.1016/j. ddmod.2004.09.008.

19. Fawcett JW, Keynes RJ. Peripheral nerve regeneration. Annu Rev Neurosci. 1990;13:43-60. doi:10.1146/annurev.neuro.13.1.43.
20. Navarro X, Vivó M, Valero-Cabré A. Neural plasticity after peripheral nerve injury and regeneration. Prog Neurobiol. 2007;82:163-201. doi:10.1016/j. pneurobio.2007.06.005.

21. Geuna S, Raimondo S, Ronchi G, Di Scipio F, Tos P, Czaja K, et al. Chapter 3: histology of the peripheral nerve and changes occurring during nerve regeneration. Int Rev Neurobiol. 2009; 87:27-46. doi:10.1016/ S0074-7742(09)87003-7.

22. Lim JY, Han TR. Effect of electromyostimulation on a apoptosis-related factors in denervation and reinnervation of rat skeletal muscles. Muscle Nerve. 2010;42(3):422-30. doi:10.1002/mus.21719.

23. Eberstein A, Pachter BR. The effect of electrical stimulation on reinnervation of rat muscle: contractile properties and endplate morphometry. Brain Res. 1986;384(2):304-10. doi:10.1016/00068993(86)91166-2.

24. Jaweed MM, Herbison GJ, Ditunno JF. Direct electrical stimulation of rat soleus during denervationreinnervation. Exp Neurol. 1982;75(3):589-99. doi:10.1016/0014-4886(82)90027-9.

25. Dow DE, Cederna PS, Hassett CA, Dennis RG, Faulkner JA. Electrical stimulation prior to delayed reinnervation does not enhance recovery in muscles of rats. Restor Neurol Neurosci. 2007;25(5-6):601-10.

26. Gigo-Benato D, Russo TL, Geuna S, Domingues NR, Salvini TF, Parizotto NA. Electrical stimulation impairs early functional recovery and accentuates skeletal muscle atrophy after sciatic nerve crush injury in rats. Muscle Nerve. 2010;41(5):685-93. doi:10.1002/ mus.21549.

27. Tomori K, Ohta Y, Nishizawa T, Tamaki H, Takekura H. Low-intensity electrical stimulation ameliorates disruption of transverse tubules and neuromuscular junctional architecture in denervated rat skeletal muscle fibers. J Muscle Res Cell Motil. 2010;31(3):195205. doi:10.1007/s10974-010-9223-8.

28. Russo TL, Peviani SM, Durigan JLQ, Gigo-Benato D, Delfino GB, Salvini TF. Stretching and electrical stimulation reduce the accumulation of myoD, myostatin and atrogin-1 in denervated rat skeletal muscle. J Muscle Res Cell Motil. 2010;31(1):45-57. doi:10.1007/ s10974-010-9203-z. 
29. Lima SC, Caierão QM, Peviani SM, Russo TL, Sommaz MC, Salvini TF, et al. Muscle and nerve responses after different intervals of electrical stimulation sessions on denervated rat muscle. Am J Phys Med Rehabil. 2009;88(2):126-35. doi:10.1097/ PHM.0b013e318186bf6c.

30. Russo TL, Peviani SM, Freria CM, Gigo-Benato D, Geuna $\mathrm{S}$, Salvini TF. Electrical stimulation based on chronaxie reduces atrogin- 1 and myoD gene expressions in denervated rat muscle. Muscle Nerve. 2007;35(1):87-97. doi:10.1002/mus.20668.

31. Dow DE, Carlson BM, Hassett CA, Dennis RG, Faulkner JA. Electrical stimulation of denervated muscles of rats maintains mass and force, but not recovery following grafting. Restor Neurol Neurosci. 2006;24(1):41-54.

32. Dow DE, Faulkner JA, Dennis RG. Distribution of rest periods between electrically generated contractions in denervated muscles of rats. Artif Organs. 2005;29(6):432-5.

33. Dow DE, Cederna PS, Hassett CA, Kostrominova TY, Faulkner JA, Dennis RG. Number of contractions to maintain mass and force of a denervated rat muscles. Muscle Nerve. 2004;30(1):77-86. doi:10.1002/ mus.20054.

34. Kanaya F, Tajima T. Effect of electrostimulation on denervated muscle. Clin Orthop Relat Res. 1992;283:296-301.

35. Tower SS. Trophic control of non-nervous tissue by the nervous system: a study of a muscle and bone innervated from an isolated and quiescent region of spinal cord. J Comp Neurol. 1937;67(2):241-67. doi:10.1002/cne.900670206.
36. Hyatt JP, Roy RR, Baldwin KM, Edgerton VR. Nerve activity-independent regulation of skeletal muscle atrophy: role of MyoD and myogenin in satellite cells and myonuclei. Am J Physiol Cell Physiol. 2003;285(5):1161-73.

37. Midrio M. The denervated muscle: facts and hypotheses. A historical review. Eur J Appl Physiol. 2006;98(1):1-21. doi:10.1007/s00421-006-0256-z.

38. Dennis RG, Dow DE, Faulkner JA. An implantable device for stimulation of denervated muscles in rats. Med Eng Phys. 2003;25(3):239-53. doi:10.1016/ S1350-4533(02)00193-5.

39. Dennis RG, Dow DE. Excitability of skeletal muscle during development, denervation and tissue culture. Tissue Eng. 2007;13(10):2395-404. doi:10.1089/ ten.2006.0367.

40. Salmons S, Ashley Z, Sutherland H, Russold MF, Li F, Jarvis JC. Functional electrical stimulation of denervated muscles: basic issues. Artif Organs. 2004;29(3):199202. doi:10.1111/j.1525-1594.2005.29034.x.

41. Nelson RM, Hayes KW, Currier DP. Eletroterapia clínica. 3a ed. São Paulo: Manole; 2003.

42. Mokrusch T, Engelhardt A, Eichhorn KF, Prischenk G, Prischenk H, Sack G, et al. Effects of long-impulse electrical stimulation on atrophy and fiber type composition of chronically denervated fast rabbit muscle. J Neurol. 1990;237(1):29-34. doi:10.1007/BF00319664.

Recebido: 24/03/2011 Received: 03/24/2011

Aprovado: 07/10/2011 Approved: 10/07/2011 